\title{
RELAÇÕES ENTRE AS DOENÇAS RESPIRATÓRIAS E A POLUIÇÃO ATMOSFÉRICA E VARIÁVEIS CLIMÁTICAS NA CIDADE DE CURITIBA, PARANÁ, BRASIL
}

\author{
Paulo Ricardo Bittencourt Guimarães ${ }^{1}$, Ricardo Berger ${ }^{2}$, Fernando Lucambio Perez ${ }^{3}$, \\ Paulo de Tarso de Lara Pires ${ }^{2}$ \\ ${ }^{1}$ Estatístico, Doutorando em Engenharia Florestal, UFPR, PR, Brasil - prbg@ufpr.br \\ ${ }^{2}$ Eng.Florestal, Dr., Depto. Economia Rural e Extensão, UFPR, Curitiba, PR, Brasil - berger@ufpr.br;ptlpires@ufpr.br \\ ${ }^{3}$ Matemático, Dr., Depto. Estatística, UFPR, Curitiba, PR, Brasil - lucambio@ufpr.br \\ Recebido para publicação: 10/10/2011 - Aceito para publicação: 05/11/2012
}

\begin{abstract}
Resumo
Neste estudo, foram observados níveis de concentrações de poluentes atmosféricos da cidade de Curitiba, no período de novembro de 2003 a junho de 2008, da estação automática de monitoramento da praça Ouvidor Pardinho. O objetivo foi desenvolver uma metodologia de modelagem estatística que explique o número de notificações de doenças respiratórias registradas em idosos (acima de 60 anos) e crianças (abaixo de 5 anos). Além das concentrações de poluentes, algumas variáveis climáticas foram analisadas. Após estudo de diversas metodologias estatísticas, considerou-se mais adequado o ajuste de Modelos Aditivos Generalizados para Locação, Escala e Forma. Os modelos ajustados apresentaram resultados satisfatórios e consideraram como variáveis significativas as partículas inaláveis e a temperatura. Além disso, verificou-se que existe redução proporcional do número de notificações de doenças respiratórias no período observado, em consequência da redução dos níveis de concentração de partículas inaláveis no município de Curitiba. Os resultados encontrados podem ter sido influenciados pelas campanhas de vacinação de idosos, pela utilização de motores menos poluentes no transporte coletivo, pelo aumento da produção de carros "flex" e também pela grande cobertura vegetal existente no município, responsável pelos mais altos índices de área verde por habitante entre os grandes centros urbanos do Brasil.

Palavras-chave: Modelos lineares generalizados; poluentes; partículas inaláveis; GAMLSS.
\end{abstract}

\begin{abstract}
Relation between respiratory diseases and air pollution and climatic variables, in Curitiba, Parana, Brazil. In this research, concentration levels of atmospheric pollutants were observed between November 2003 and June 2008 from an automated monitoring station located at Ouvidor Pardinho Square, City of Curitiba. It aimed to develop a methodology of statistical model in order to explain the number of notifications of respiratory diseases registered in the elderly (above the age of 60 years) and children (below the age of 5 years). Apart from the concentration of pollutants, climatic variables were also analyzed. After study of several statistical methodologies, it was considered that the most adequate was an adjustment of the Generalized Additive Models for Location, Scale and Shape. The adjusted models presented satisfactory results and considered as significant variables the particulate matter and temperature. It was also observed a proportional reduction in the number of notifications of respiratory diseases within the observed period as a consequence of the concentration level reduction of the particulate matter in the city. These results may have been influenced by vaccination campaigns involving the elderly, by use of less polluting engines in the public transportation system, by an increase in production of "flex"- type cars and also by the large vegetation coverage in the city, which is responsible for one of the highest indexes of green area per inhabitant amongst the large urban centers in Brazil.

Keywords: Generalized linear models; pollutants; particulate matter; GAMLSS.
\end{abstract}

\section{INTRODUÇÃO}

A poluição do ar tem sido um tema extensivamente pesquisado nas últimas décadas e caracteriza-se como um fator de grande importância na busca da preservação do meio ambiente e na implementação de um desenvolvimento sustentável, pois seus efeitos afetam de diversas formas os materiais, os ecossistemas e, principalmente, a saúde humana. 
$\mathrm{Na}$ cidade de Curitiba, esse assunto poucas vezes recebe destaque na mídia. Os responsáveis pelo monitoramento da poluição afirmam que em $90 \%$ das medições a qualidade do ar é boa. Não se sabe, porém, se esse índice é suficiente para não afetar a população, principalmente crianças e idosos.

A cidade de Curitiba, capital do estado do Paraná, Brasil, apesar de seu planejamento, não escapa dos problemas advindos de um alto crescimento populacional. A camada de ar que recobre a cidade é afetada pelo crescimento que a cidade vem apresentando. Além da natural expansão da ocupação do solo urbano para os limites territoriais do município, a partir da década de 80 , o adensamento populacional tem se manifestado notadamente pela verticalização de suas construções ao longo das principais vias de circulação da cidade, denominadas eixos estruturais, que assim podem atuar como verdadeiras barreiras à livre circulação do ar (DANNI-OLIVEIRA, 2002).

Manifestando-se no cotidiano dos habitantes da cidade por meio de frequentes engarrafamentos de tráfego, o expressivo aumento da frota de veículos vem somar-se às evidências de crescimento da cidade e constituir-se numa das principais fontes de poluentes do ar. Com uma frota de mais de 1 milhão de veículos em 2008, segundo o Departamento de Trânsito do Paraná (DETRAN-PR), transitando em suas ruas, a poluição, muitas vezes, tende a ser particularmente acentuada nos setores dos eixos estruturais, onde se deu elevado adensamento vertical.

Mais recentemente, considerando-se as atuais políticas econômicas adotadas pelas administrações estadual e municipal de incentivar a instalação de indústrias nos limites da Região Metropolitana de Curitiba, têm-se criado feições urbanas que parecem comprometer a qualidade de seu ar, apesar do conceito de "cidade ecológica" que a mídia e os órgãos municipais e estaduais têm propalado com êxito.

Assim, em ocasiões de falta de vento e chuva, a qualidade do ar pode ficar comprometida, uma vez que a estrutura topográfica edificada tende a propiciar o confinamento dos poluentes lançados pelo intenso trânsito de veículos automotores que nela trafegam.

O objetivo deste trabalho é avaliar a relação entre a poluição atmosférica e variáveis climáticas com o número de casos de doenças respiratórias na população infantil e idosa no município de Curitiba, Paraná. Para isso, foi observado o comportamento da incidência de doenças respiratórias na população da cidade de Curitiba no período de 2003 a 2008. Desenvolveu-se uma metodologia estatística de modelagem adequada aos dados e estimou-se o modelo mais adequado para explicar o comportamento das notificações de doenças respiratórias em idosos e crianças e a sua relação com concentrações de poluentes e variáveis climáticas.

\section{Utilização de métodos estatísticos}

No artigo de Conceição et al. (2001), que cita estudo realizado na cidade de São Paulo (SP), são apresentados Modelos Lineares Generalizados (MLG) e Modelos Aditivos Generalizados (MAG) para a análise da associação entre poluição atmosférica e marcadores de morbi-mortalidade.

Freitas et al. (2004) investigaram os efeitos de curto prazo da poluição atmosférica na morbidade respiratória de menores de 15 anos e na mortalidade de idosos na cidade de São Paulo. Foi utilizada para análise a regressão de Poisson em modelos MAG. Os modelos foram ajustados para efeitos da tendência temporal, sazonalidade, dias da semana, fatores meteorológicos e autocorrelação.

O artigo de Bakonyi et al. (2004) é um estudo ecológico de séries temporais realizado no município de Curitiba, estado do Paraná. Os dados diários dos atendimentos ambulatoriais se referem aos atendimentos de crianças na faixa etária de 0 a 14 anos, no período de $1^{\circ}$ de janeiro de 1999 a 31 de dezembro de 2000.

Nascimento et al. (2006) realizaram um estudo ecológico de séries temporais na cidade de São José dos Campos(SP) nos anos de 2000 e 2001. Também foi utilizada a modelagem MAG de regressão Poisson.

Chiogna e Gaetan (2002) propuseram uma modelagem dos efeitos da exposição a poluentes na cidade de Birmingham, Alabama, usando Modelos Lineares Generalizados Dinâmicos (MLGD).

Dethlefsen e Christensen (2006) formularam procedimentos de ajustes utilizando a modelagem de "espaço estados" com variável resposta seguindo distribuição dentro da família exponencial. Desenvolveram a biblioteca SSPIR implementada no software $R$ para estimação de parâmetros variando ao longo do tempo, fazendo uso da metodologia filtro de Kalman.

Rigby et al. (2008) desenvolveram uma metodologia própria para ajuste de dados superdispersos, especificamente para dados de contagem, usando a distribuição Gauss-Poisson inversa 
generalizada. Criaram a biblioteca GAMLSS implementada no software R.

Em estudos em que o objetivo é avaliar os efeitos da poluição atmosférica sobre a saúde dos habitantes de grandes centros urbanos, a variável resposta, nesses estudos, geralmente é alguma contagem de eventos que representam danos à saúde, como o número de óbitos ou o número de internações por determinada causa respiratória. A escolha da concentração de alguns gases, como óxidos de nitrogênio $\left(\mathrm{NO}_{\mathrm{x}}\right)$, dióxido de enxofre $\left(\mathrm{SO}_{2}\right)$, monóxido de carbono $(\mathrm{CO})$ ou partículas inaláveis (PI), como candidatos a variáveis explicativas também é bastante comum. Além disso, há evidências significativas de que fatores sazonais e condições climáticas, como a temperatura e a umidade do ar, influenciam significativamente o fenômeno.

\section{MATERIAL E MÉTODOS}

\section{Monitoramento em Curitiba}

A Secretaria Estadual de Meio Ambiente (SEMA), através do Instituto Ambiental do Paraná (IAP), faz o monitoramento da qualidade do ar em Curitiba desde a década de 80. Em 2008, o instituto era responsável por 11 estações de monitoramento, sendo quatro em Curitiba e sete em Araucária. Dessas estações, três automáticas localizadas em Curitiba (Santa Cândida, Boqueirão e praça Ouvidor Pardinho), outras quatro automáticas em Araucária; e mais quatro estações manuais, uma em Curitiba e três em Araucária.

Cada estação possui instrumentos que analisam poluentes atmosféricos e parâmetros meteorológicos. O equipamento das estações manuais opera com o auxílio de filtros, cuja análise é realizada posteriormente em laboratório. As estações automáticas operam com analisadores que fazem a coleta e análise dos poluentes simultaneamente. Os resultados são armazenados por um sistema computadorizado, obtendo-se as médias horárias dos poluentes. Entre os indicadores analisados estão as partículas totais em suspensão (PTS), a fumaça, as partículas inaláveis ( $\mathrm{PI}$ ou $\left.\mathrm{PM}_{10}\right)$, o dióxido de enxofre $\left(\mathrm{SO}_{2}\right)$, o monóxido de carbono (CO), o ozônio $\left(\mathrm{O}_{3}\right)$ e o dióxido de nitrogênio $\left(\mathrm{NO}_{2}\right)$ (IAP, 2008).

\section{Descrição da região em estudo}

A cidade de Curitiba tem uma área de $430,9 \mathrm{~km}^{2}$, fica no centro da Região Metropolitana de Curitiba (RMC), que recobre 26 municípios e conta com uma população de 1.746.896 habitantes (IBGE, 2010), apresentando uma taxa de crescimento populacional de $3,1 \%$ ao ano.

Está localizada no primeiro planalto do estado do Paraná e tem um clima temperado marítimo ou clima subtropical de altitude ( $\mathrm{Cfb}$ ), de acordo com a classificação climática de Köppen. Os invernos são brandos, com temperatura média de $13{ }^{\circ} \mathrm{C}$ no mês mais frio, caindo por vezes abaixo de $2{ }^{\circ} \mathrm{C}$ em dias mais frios. Apresenta geadas ocasionais e temperaturas mínimas de cerca de $-3{ }^{\circ} \mathrm{C}$. Durante o verão, a temperatura média é em torno de $21^{\circ} \mathrm{C}$, mas pode subir acima de $30^{\circ} \mathrm{C} \mathrm{em}$ dias mais quentes, quando são registradas temperaturas de até $35^{\circ} \mathrm{C}$. Ondas de calor durante o inverno e ondas de frio no verão não são incomuns, e mesmo dentro de um único dia pode haver uma grande variação, uma característica típica do clima subtropical (IPPUC, 2002).

A umidade relativa varia entre 75 e $85 \%$ (média mensal). As precipitações ocorrem durante o ano inteiro, com maior intensidade nos meses de verão (dezembro até fevereiro) e um pouco menos no inverno (junho até agosto). Na média são registradas chuvas de $150 \mathrm{~mm} / \mathrm{mês} \mathrm{no} \mathrm{verão} \mathrm{e} 80 \mathrm{~mm} / \mathrm{mês} \mathrm{no}$ inverno (IPPUC, 2002). Os ventos vêm geralmente do leste, segundo as estações de monitoramento da qualidade do ar.

\section{Descrição dos dados}

Neste estudo se pretende relacionar os efeitos de diferentes poluentes e a saúde dos habitantes de Curitiba, através do ajuste de um modelo de regressão. Para isso, foram observadas, especificamente, as populações mais suscetíveis, as faixas etárias de idosos e crianças. Assim, pretende-se relacionar o número de casos de doenças respiratórias na população infantil e idosa com as concentrações de gases poluentes na atmosfera e algumas variáveis meteorológicas. As doenças respiratórias consideradas neste estudo estão definidas na Classificação Internacional de Doenças, volume 10 (CID-10).

A variável resposta, em ambos os casos, é uma contagem de eventos que representam danos à saúde, nesse caso, o número de notificações de doenças respiratórias em crianças e idosos, agrupados 
semanalmente durante o período 2003-2008 (SMS). Os dados correspondem a 95,2\% de casos de residentes na cidade de Curitiba, na rede pública, segundo a SMS.

Os dados são agrupados por semana epidemiológica e se referem ao total de notificações de doenças respiratórias em hospitais e postos de saúde no município de Curitiba (rede pública). As faixas etárias consideradas são crianças até 5 anos e idosos acima de 60 anos. Por convenção internacional, as semanas epidemiológicas são contadas de domingo a sábado, sendo que a primeira semana do ano é aquela que contém o maior número de dias de janeiro e a última a que contém o maior número de dias de dezembro.

As concentrações de gases poluentes são as possíveis variáveis explicativas. Essas concentrações têm medição diária nas estações de monitoramento de Curitiba, no período 2003-2008 (fornecidos pelo IAP).

Cada poluente foi padronizado segundo critérios da resolução 03/90 do Conselho Nacional do Meio Ambiente (CONAMA). O objetivo da padronização é deixar todas as substâncias em uma única escala.

Algumas variáveis meteorológicas foram também consideradas (temperatura e umidade relativa do ar), e os dados foram fornecidos pelo Instituto Tecnológico Simepar (Sistema Meteorológico do Paraná).

Todas as informações foram agrupadas por semana epidemiológica, para se ajustarem aos dados das notificações de doenças respiratórias. Ou seja, como as medidas de concentrações de poluentes e as medidas meteorológicas são medidas diariamente, foi necessário calcular as médias semanais referentes aos mesmos períodos dos registros de notificações de doenças respiratórias, obtidos pela SMS.

Como fonte de coleta de dados das concentrações de poluentes, optou-se, entre as estações de monitoramento da qualidade do ar, pela utilização dos dados da estação automática da praça Ouvidor Pardinho, na região central de Curitiba, por representar informações mais consistentes.

\section{Modelos Lineares Generalizados}

Metodologias para modelagem de dados na forma de proporções e de contagem foram propostas antes da década de 70 (GRIZZLE et al., 1969), sendo que a unificação do procedimento de modelagem foi proposta por Nelder e Wedderburn (1972), sob o nome de Modelos Lineares Generalizados. Englobam os modelos de regressão linear simples e múltipla, regressão logística, regressão de Poisson e muitos outros, como modelos log-lineares para dados categorizados.

A seleção de modelos é uma parte importante de toda pesquisa, envolvendo a procura de um modelo o mais simples possível, razoável, que descreva bem os dados observados. Entretanto, no mundo real, muitas vezes é necessário analisar dados não normais. No caso em que as observações assumem distribuições da família exponencial, os modelos lineares generalizados são bastante adequados. Uma família exponencial uniparamétrica é um conjunto de distribuições de probabilidade, cuja função densidade de probabilidade (ou função de probabilidade) pode ser expressa da seguinte forma:

$$
f_{\underline{x}}(\underline{x} ; \theta)=h(\underline{x}) \exp (\eta(\theta) T(\underline{x})-A(\theta))
$$

Em que: $\mathrm{T}(\underline{x}), \mathrm{h}(\underline{x}), \eta(\theta)$ e $\mathrm{A}(\theta)$ são funções conhecidas;

$\theta$ é o parâmetro da família;

$\underline{x}$ é um vetor de observações;

Se $\eta(\theta)=\theta$, então diz-se que a família exponencial está na forma canônica.

$\mathrm{T}(\underline{x})$ é uma estatística suficiente para o parâmetro da distribuição. Assim, para famílias exponenciais, existe uma estatística suficiente cuja dimensão é igual ao número de parâmetros a serem estimados;

$\eta$ é chamado de parâmetro natural. O conjunto de valores de $\eta$ para os quais a função $f_{X}(\underline{x} ; \theta)$ é finita é chamado de espaço paramétrico natural;

$A(\theta)$ é um fator de normalização ou função de $\log$-partição, sem a qual $f_{X}(\underline{x} ; \theta)$ não seria uma distribuição de probabilidade. 
Os elementos básicos desses modelos são:

1. $\mathrm{K}$ valores independentes $\mathrm{Y}_{1}, \ldots, \mathrm{Y}_{\mathrm{K}}$, de uma variável resposta que segue uma distribuição da família exponencial, com valor esperado $\mathrm{E}\left(\mathrm{Y}_{\mathrm{i}}\right)=\mu_{\mathrm{i}}$;

2. $\mathrm{K}$ vetores $\underline{x}_{\mathrm{i}}=\left(\begin{array}{lll}1 & \mathrm{x}_{\mathrm{i} 1} \mathrm{x}_{12} \ldots \mathrm{x}_{\mathrm{ip}}\end{array}\right)^{\mathrm{t}}, \mathrm{i}=1, \ldots, \mathrm{K}$, contendo os valores das $\mathrm{p}$ variáveis explicativas;

3. Uma função monotônica e diferenciável $g$, chamada de função de ligação, tal que $g\left(\mu_{\mathrm{i}}\right)=\underline{x}_{\mathrm{i}}{ }^{\prime} \beta, \mathrm{i}=1, \ldots$, $\mathrm{K} \operatorname{com} \beta=\left(\beta_{1} \beta_{2} \ldots \beta_{\mathrm{p}}\right)$ representando o vetor de parâmetros a serem estimados.

Se g é a função identidade, isto é, se $\mathrm{g}\left(\mu_{\mathrm{i}}\right)=\mu_{\mathrm{i}}$, então $\mu_{\mathrm{i}}=\mathrm{E}\left(\mathrm{Y}_{\mathrm{i}}\right)=\underline{x}_{\mathrm{i}}{ }^{\prime} \beta$, e o modelo resultante, com algumas suposições adicionais, é o modelo de regressão linear gaussiana citado anteriormente.

$\mathrm{O}$ vetor de parâmetros $\beta$ pode ser estimado pelo método de máxima verossimilhança, e os cálculos envolvem um procedimento iterativo.

Nelder e Wedderburn (1972) mostraram, então, que a maioria dos problemas estatísticos, que surgem nas áreas de agricultura, demografia, ecologia, economia, geografia, geologia, história, medicina, ciência política, psicologia, sociologia, zootecnia etc., podem ser formulados, de uma maneira unificada, como modelos de regressão. Esses modelos envolvem uma variável resposta univariada, variáveis explicativas e uma amostra aleatória de n observações, sendo que:

- A variável resposta, componente aleatório do modelo, tem uma distribuição pertencente à família exponencial na forma canônica (distribuições normais, gama e normal inversa para dados contínuos; binomial para proporções; Poisson e binomial negativa para contagens);

- As variáveis explicativas entram na forma de um modelo linear (componente sistemático);

- A ligação entre os componentes aleatórios e sistemáticos é feita através de uma função (por exemplo, logarítmica para os modelos log-lineares).

\section{Modelos Aditivos Generalizados Para Locação, Escala e Forma (GAMLSS)}

Foram introduzidos por Rigby e Stasinopoulos $(2001,2005)$ e Akantziliotouet al. (2002) como uma maneira de resolver os problemas advindos das limitações dos Modelos Lineares Generalizados (NELDER; WEDDERBURN, 1972) e Modelos Aditivos Generalizados (HASTIE; TIBSHIRANI, 1990).

No modelo GAMLSS, a suposição de que a distribuição da variável resposta (y) pertença à família exponencial é flexibilizada e substituída por uma família de distribuições geral, incluindo distribuições altamente assimétricas e/ou com problemas de curtose. A parte sistemática do modelo é expandida, para permitir a modelagem não somente da média (locação), mas de outros parâmetros da distribuição de y como funções de parâmetros lineares e/ou aditivos não paramétricos das variáveis explanatórias e/ou efeitos aleatórios. Estimação de máxima-verossimilhança (penalizada) é usada para ajustar os modelos.

Portanto, GAMLSS é especialmente desenvolvido para modelar uma variável resposta que não segue uma distribuição da família exponencial (por exemplo, dados com distribuição leptocúrtica ou platicúrtica e/ou com assimetria positiva ou negativa, ou contagens superdispersas) ou que exibem heterogeneidade (por exemplo, onde a escala ou forma da distribuição da variável resposta muda dependendo das variáveis explanatórias).

Se a variável resposta é uma contagem, muito provavelmente a distribuição Poisson não irá se ajustar perfeitamente. GAMLSS fornece uma variedade de distribuições discretas (incluindo a binomial negativa) que podem ser ajustadas.

Neste estudo, ajustou-se aos dados a distribuição Gauss-Poisson Inversa Generalizada. Essa distribuição se ajusta bem aos dados de contagem, que teriam teoricamente uma distribuição Poisson, mas que não se ajustam de forma adequada por não respeitar certos pressupostos. A distribuição Poisson prevê que a média seja igual à sua variância, o que muitas vezes pode não ocorrer em dados de contagem.

Para escolha do modelo que melhor se ajusta aos dados, utilizou-se o Critério de Informação de Akaike (AIC), em conjunto com o Critério Bayesiano de Schwartz (SBC).

\section{RESULTADOS}

Verificou-se, através de análise de autocorrelação (função de autocorrelação e função de autocorrelação parcial), que não é evidente a dependência entre as observações. Esse fato se justifica porque as observações estão agrupadas por semana epidemiológica, o que, de certa forma, dissipa boa 
parte dos efeitos de autocorrelação.

Assim, utilizando a mesma biblioteca GAMLSS, ajustou-se um modelo linear generalizado, considerando que a variável resposta tem uma distribuição Gaus-Poisson Inversa Generalizada. Com essa metodologia, o ajuste obtido foi bastante satisfatório, segundo os resultados da análise de resíduos.

Foi definida no modelo uma variável "offset" -número total de notificações -, fazendo com que os valores estimados se refiram a uma proporção de casos de doenças respiratórias em relação ao total de casos.

O programa para realizar o ajuste de dados foi o software estatístico R versão 2.7.2, utilizando-se a biblioteca GAMLSS.

Na figura 1, é apresentada a frequência relativa das notificações de doenças respiratórias em relação ao total de notificações (por todos os diagnósticos), para idosos e crianças na cidade de Curitiba.

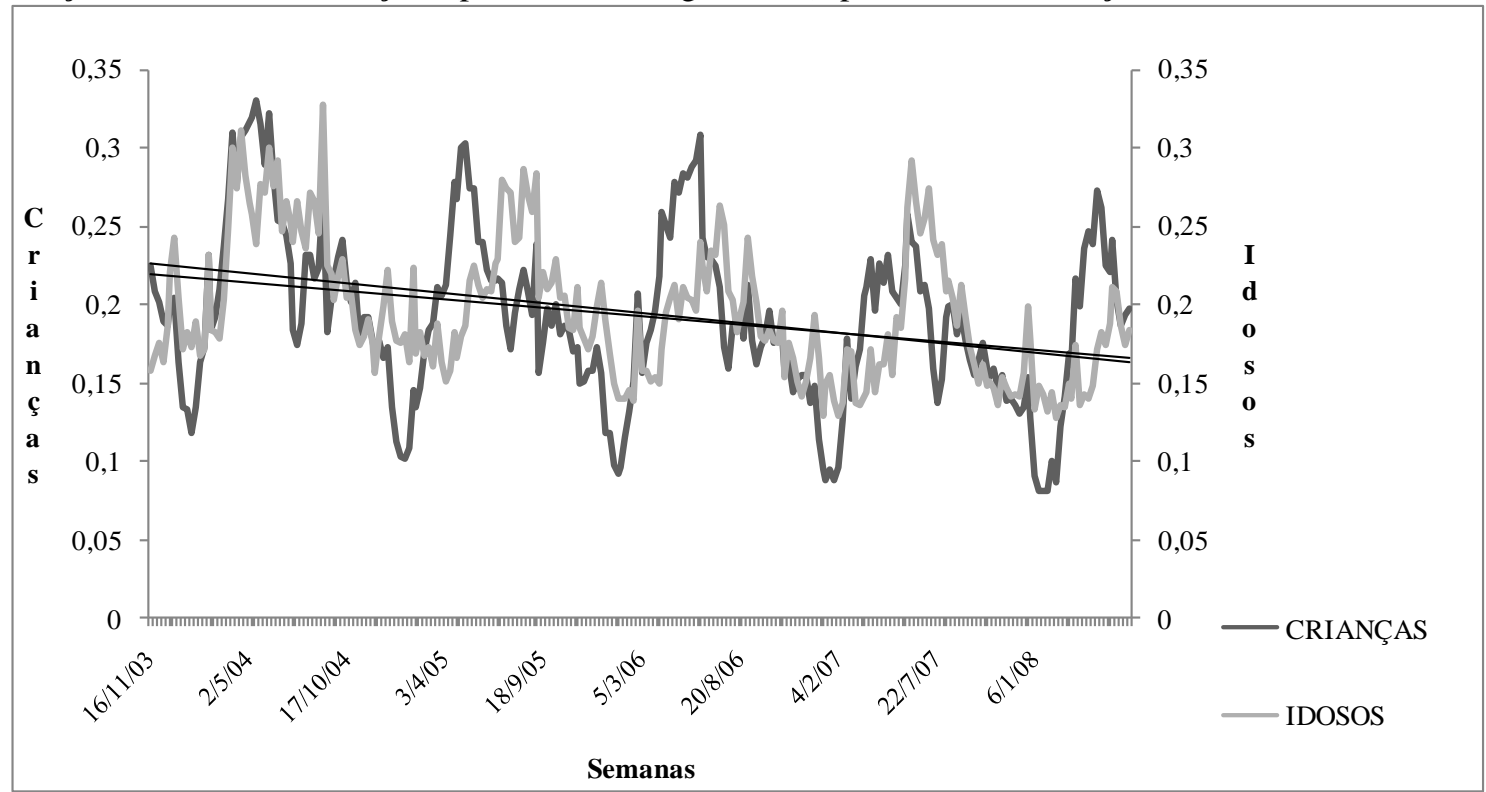

Figura 1. Séries temporais do número de notificações de doenças respiratórias em idosos e crianças e número de notificações totais - Curitiba 2003-2008.

Figure 1. Time series of the number of reports of respiratory diseases in the elderly and children and total number of notifications - Curitiba 2003-2008.

Pode-se observar que, nos dois casos, existe uma queda da representação das doenças respiratórias, tanto em idosos como em crianças. Pode-se concluir que, considerando o aumento populacional dos últimos anos, na cidade de Curitiba, existe uma redução proporcional no número de notificações de doenças respiratórias, tanto em idosos como em crianças.

A figura 2 apresenta a relação entre as notificações de doenças respiratórias e de temperatura média semanal.

Pode-se notar que existe uma visível influência da temperatura no número de notificações, tanto para idosos como para crianças. Essa relação é inversa, ou seja, para baixas temperaturas, existe um crescimento do número de notificações de doenças respiratórias, e vice-versa.

A figura 3 mostra que a concentração de partículas inaláveis possui uma relação forte (coeficiente de Spearman acima de 0,9 ) com o número de notificações de doenças respiratórias, tanto em crianças como em idosos.

Observa-se que os períodos de concentrações mais altas desse poluente coincidem com os períodos de maior número de notificações de doenças respiratórias, e vice-versa.

A figura 4 representa a tendência verificada no período em estudo, para o comportamento da concentração de matéria particulada em Curitiba. 

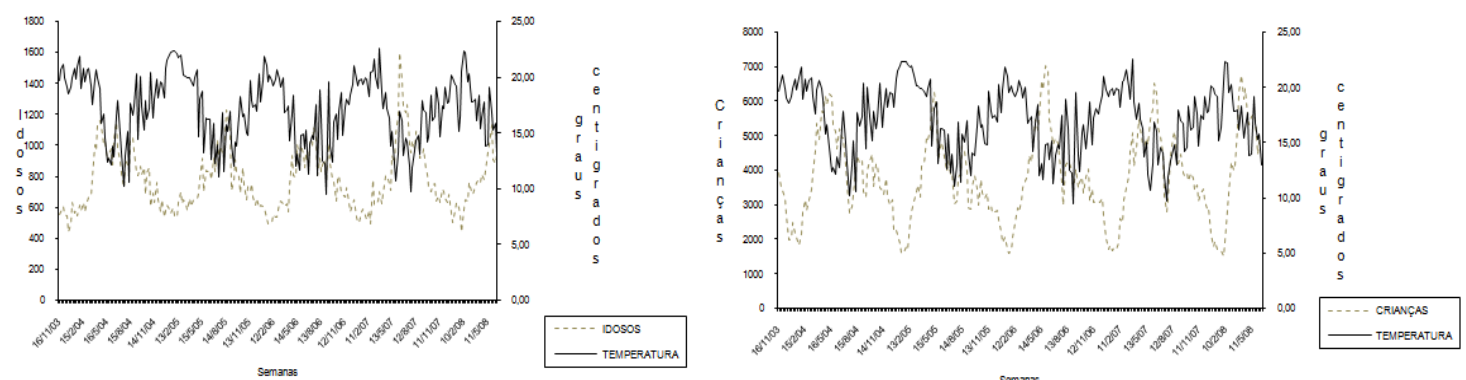

Figura 2. Relação entre o número de notificações de doenças respiratórias em idosos e crianças e temperatura.

Figure 2. Relationship between the number of reports of respiratory diseases in the elderly and children and temperature.
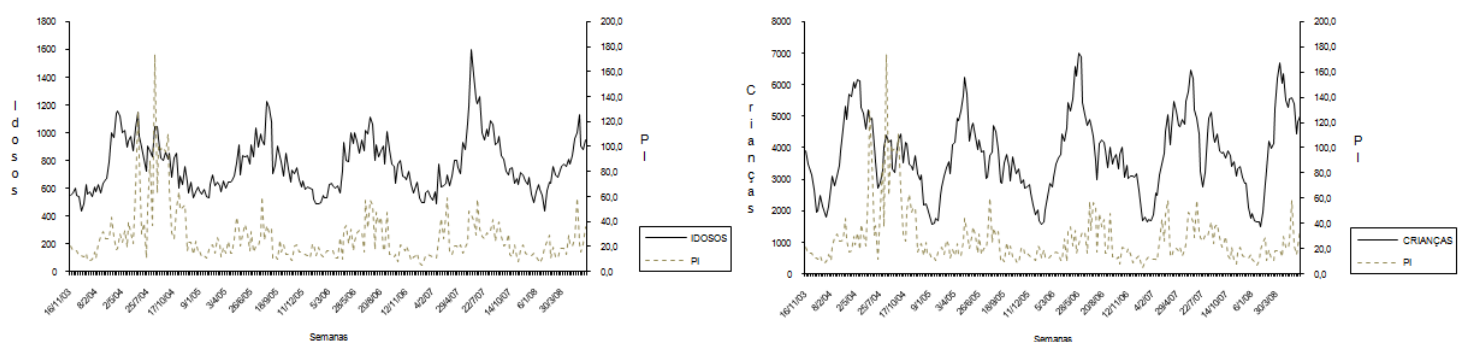

Figura 3. Relação entre número de notificações de doenças respiratórias em idosos e crianças e partículas inaláveis (PI).

Figure 3. Relation between the number of reports of respiratory diseases in the elderly and children and inhalable particles.

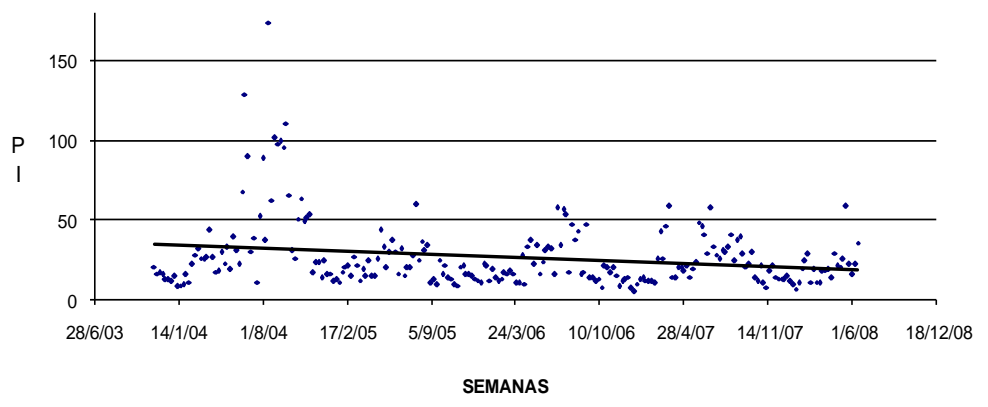

Figura 4. Partículas inaláveis (PI): tendência.

Figure 4. Particulate matter (MP10): tendency.

A seguir são apresentados os resultados dos melhores ajustes encontrados através da aplicação do método Rigby e Stasinopoulos usando o aplicativo R (biblioteca GAMLSS).

O modelo final ajustado tanto para idosos como para crianças tem a seguinte estrutura:

$$
e^{N I}=\beta_{0}+\beta_{1} T M+\beta_{\mathbf{2}} P I+\text { off } \operatorname{set}(\log (\text { NOTTOT }))
$$

Em que: NI: número de notificações de doenças respiratórias em idosos;

TM: temperatura média;

PI: material particulado;

NOTTOT: número total de notificações. 
Dessa forma, o modelo final para idosos (1), após exclusão das variáveis não significativas, é:

\begin{tabular}{|c|c|c|c|c|}
\hline & Estimativa & Erro Padrão & $\mathrm{t}$ & $\mathrm{p}$-valor \\
\hline Intercepto & $-3,890$ & 0,074 & $-52,62$ & $9,904 \mathrm{e}-133$ \\
\hline TM & $-0,029$ & 0,004 & $-7,144$ & $1,107 \mathrm{e}-11$ \\
\hline PI & 0,004 & 0,0005 & 8,877 & $1,726 \mathrm{e}-16$ \\
\hline
\end{tabular}

Assim, das variáveis explicativas alocadas inicialmente ao modelo, apenas a temperatura e o material particulado afetam o número de notificações de doenças respiratórias em idosos.

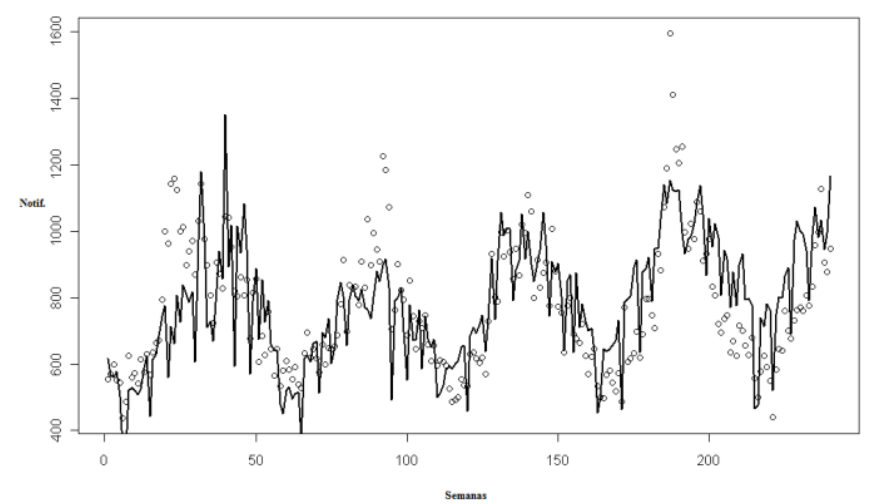

Figura 5. Modelo ajustado para as notificações de doenças respiratórias em idosos na cidade de Curitiba. Figure 5. Adjusted model for notifications of respiratory diseases in the elderly in the city of Curitiba.

O modelo final para crianças (2), após exclusão das variáveis não significativas, é:

\begin{tabular}{|c|c|c|c|c|}
\hline & Estimativa & Erro Padrã & $\mathrm{t}$ & p-valor \\
\hline ept & $-2,192$ & 0,103 & $-21,221$ & $1,021 \mathrm{e}-56$ \\
\hline TM & $-0,036$ & 0,005 & $-6,514$ & $4,330 \mathrm{e}-10$ \\
\hline PI & 0,004 & 0,00076 & 5,742 & $2,845 \mathrm{e}-08$ \\
\hline
\end{tabular}

Novamente, apenas a temperatura e o material particulado afetam significativamente o número de notificações de doenças respiratórias em crianças.

\section{DISCUSSÃO}

Os resultados apontam uma redução do número de notificações de doenças respiratórias entre os idosos, pela diminuição das concentrações de partículas inaláveis. Outra possível razão para essa redução de notificações, apontada pela Secretaria Municipal de Saúde, é a campanha de vacinação antigripe. Nichol et al. (2007) avaliaram o efeito da vacinação antigripe em idosos durante 10 anos em três regiões americanas diferentes. Eles demonstraram que a vacinação foi associada a uma diminuição média de $27 \%$ no risco de hospitalização por pneumonia ou influenza no inverno e a uma redução de $48 \%$ no risco de morte por qualquer causa.

Entre os idosos, alguns estudos demonstram que a vacinação pode reduzir entre 32 e $45 \%$ o número de hospitalizações por pneumonias e em 39 a $75 \%$ a mortalidade global. Entre os residentes em lares, pode reduzir o risco de pneumonia em, aproximadamente, $60 \%$, e o risco global de hospitalização e morte em cerca de 50\% e 68\%, respectivamente (ALLSUP et al. (2004); WANG e WANG (2004)), referem ainda a redução de mais de 50\% nas doenças relacionadas à influenza. 


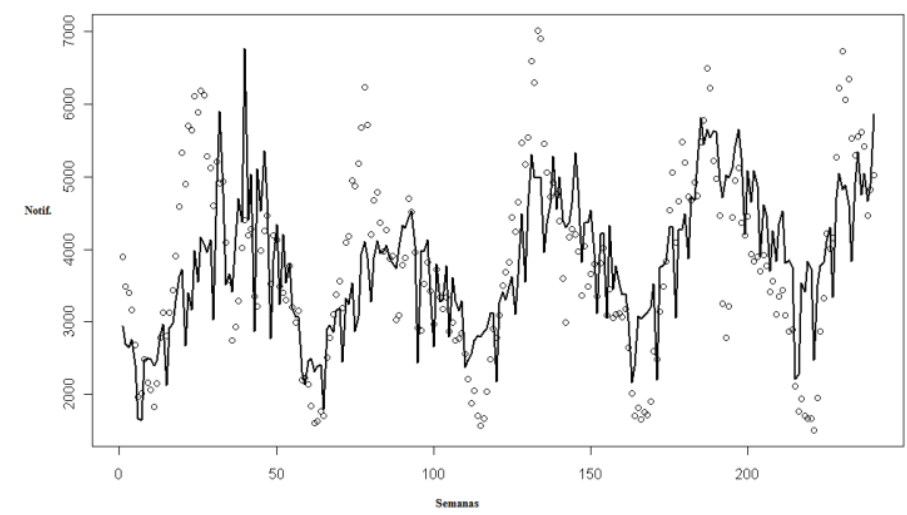

Figura 6. Modelo ajustado para as notificações de doenças respiratórias em crianças na cidade de Curitiba.

Figure 6. Adjusted model for notifications of respiratory diseases in children in the city of Curitiba.

O fato de a variável temperatura se mostrar significativa no modelo ajustado já era esperado. Demonstra que a temperatura ainda é um dos fatores mais explicativos da ocorrência de doenças respiratórias, e mesmo que se tenha uma série de ações governamentais, a queda de temperatura ainda assim afetará os sistemas cardiorrespiratórios de crianças e idosos.

Contudo, a redução dos níveis de pobreza com certeza possibilita e continuará possibilitando à população menos privilegiada um melhor atendimento por parte do sistema público de saúde municipal e assim melhor se defender dos efeitos térmicos sazonais da cidade.

Analisando o quanto a poluição custa ao país, é importante destacar que a saúde no Brasil apresentou mudanças expressivas nas últimas décadas, com incrementos na expectativa de vida, redução da mortalidade infantil, aumento de cobertura em programas de imunização e erradicação de doenças imunopreventivas e incorporação de tecnologias de ponta e resolutivas. Todas essas transformações evoluíram com efeitos profundos nos indicadores epidemiológicos e econômicos do país.

Entretanto, segundo relatório da Organização Mundial da Saúde (OMS, 2004), o Brasil figura entre os países com maior população, mas com recursos em saúde muito inferiores aos descritos em países desenvolvidos. Em 2002, foram alocados 7,8\% do produto interno bruto no setor da saúde, sendo $46 \%$ de fonte governamental. Os gastos em saúde, por habitante, foram em média de US\$206, muito inferiores ao despendido por outros países, como Canadá (US\$2.222), Reino Unido (US\$2.031), Estados Unidos (US\$ 5.274) ou mesmo Uruguai (US\$ 361). Aproximadamente 75\% da população brasileira é dependente exclusivamente do sistema público de atenção à saúde. Em 2003, as despesas com ações e serviços públicos de saúde foram de $\mathrm{R} \$ 53,6$ bilhões pelas três esferas governamentais, correspondendo a $\mathrm{R} \$ 303,17$ por habitante. A alocação de verbas no setor desaúde em termos relativos não teve incrementos significativos nos últimos anos, embora as necessidades e demandas cresçam exponencialmente.

Em consulta aobanco de dados do Sistema Único de Saúde (SUS), em 12/07/2009, o custo médio de internação por doença respiratória foi de aproximadamente $\mathrm{R} \$ 650,00$ no período de 2005 a 2008 , em Curitiba. Considerando a totalidade de internamentos realizados nesse período, isso representa um gasto bastante significativo entre os gastos do SUS. A redução de casos de doenças respiratórias significa a possibilidade de atendimento maior em outras doenças. Isso pode ser atingido pela redução dos índices de poluição de Curitiba, como demonstrado neste trabalho.

Interpretando os modelos ajustados, observa-se a importância de se continuar investindo na redução das concentrações de partículas inaláveis. Para ilustrar, suponha-se a temperatura estável em 17,1 graus (média do período): a redução de 10 unidades no índice da concentração de partículas inaláveis implicará uma redução de 3,9\% no número de notificações de doenças respiratórias, tanto em idosos como em crianças. Em um ano, isso significa 1.596 notificações a menos em idosos e 7.874 a menos em crianças.

Uma das principais explicações para a redução de concentrações de poluentes diz respeito ao 
transporte coletivo da cidade de Curitiba. Os índices de poluição pelo transporte coletivo em Curitiba caíram 50\% em 2009, o equivalente a duas toneladas a menos de fumaça tóxica e material particulado no ar, por dia, mesmo com a entrada em circulação de 295 novos ônibus. Isso representa, em um ano, a redução de 700 toneladas de poluição. A redução se deve ao uso de motores eletrônicos, que equipam os ônibus do transporte urbano da capital desde 2004, atendendo às resoluções do Conama e ao Programa de Controle da Poluição do Ar por Veículos Automotores -Proconve P-5(OEI, 2006). Curitiba foi a primeira cidade do Brasil a cumprir a legislação.

Somam-se a isso o fato de a renovação da frota de veículos proporcionar uma sensível diminuição da emissão de poluentes e a melhoria da qualidade dos combustíveis, principalmente, do óleo diesel.

\section{CONCLUSÕES}

- Os modelos (1) e (2) ajustados para as notificações de doenças respiratórias em crianças e idosos na cidade de Curitiba apontam que a temperatura e a concentração de matéria particulada influenciam significativamente a variável resposta.

- Pelo comportamento das séries de notificações de doenças respiratórias, pode-se observar que existe uma redução proporcional do número de notificações, tanto em idosos como em crianças, se comparado com a tendência crescente do número de notificações por todos os diagnósticos.

- Tanto paracrianças como para idosos, identificou-se que a temperatura é uma variável que interfere significativamente no número de notificações de doenças respiratórias. O coeficiente associado à temperatura foi estimado como sendo um valor negativo, o que representa uma relação inversa, ou seja, quando há redução de temperatura, existe um aumento do número de notificações.

- As concentrações de partículas inaláveis afetam significativamente o número de notificações de doenças respiratórias, tanto em crianças como em idosos. O coeficiente estimado para essa variável é positivo, ou seja, à medida que aumenta a concentração de partículas inaláveis na atmosfera, aumenta o número de notificações por doença respiratória.

- No período em estudo, o comportamento da concentração de partículas inaláveis em Curitiba detectou que, nos últimos anos, existe uma tendência de redução significativa dos níveis. Como o modelo aponta a relação significativa entre as notificações de doenças respiratórias e a concentração de matéria particulada, esta tendência de redução da concentração é mais uma explicação para a redução do número de notificações.

\section{REFERENCIAS}

AKANTZILIOTOU, K.; RIGBY, R. A.; STASINOPOUlOS, D. M. The R implementation of generalized additive models for location, scale and shape in statistical modelling in society. Proceedings of the 17th international workshop on statistical modelling, Chania, p. 75 - 83, 2002.

ALLSUP, S.; HAYCOX, A.; REGAN, M.; GOSNEY, M. Is influenza vaccination cost effective for healthy people between ages 65 and 74 years? Vaccine, Rochester, v. 23, n. 5, p. 639 - 645, 2004.

BAKONYI, S. M. C.; OLIVEIRA, I. M. D.; MARTINS, L. C.; BRAGA, A. L. F. Poluição atmosférica e doenças respiratórias em crianças na cidade de Curitiba, PR. Revista de Saúde Pública, São Paulo, v. 38, p. 695 - 700, 2004.

CHIOGNA, M.; GAETAN, C. Dynamic generalized linear models with application to environment epidemiology. JRSS, Londres, v. 51, p. 453 - 458, 2002.

CONCEIÇÃO, G. M. S.; SINGER, JÚLIO M.; SALDIVA, P. H. N. Modelos MLG e MAG para análise da associação entre poluição atmosférica e marcadores de morbi-mortalidade: uma introdução baseada em dados da cidade de São Paulo. Brazilian Journal of Epidemiology, Brasil, v. 45, p. 206 - 219, 2001.

CONSELHO NACIONAL DO MEIO AMBIENTE (CONAMA), 1990, Resolução CONAMA 003, de 28/06/1990. Diário Oficial da União, Brasília, DF, 22/08/1990. 
DANNI OLIVEIRA, I. M. Considerações sobre a poluição do ar em Curitiba-PR face a seus aspectos de urbanização. Ra'ega, Curitiba, n. 4, p. 101 - 110, 2002.

DETHLEFSEN, C.; LUNDB YE-CHRISTENSEN, S. Formulating state space models in $r$ with focus on longitudinal regression models. Journal of Statistical Software, Los Angeles, v. 16, n. 1, 2006.

DEPARTAMENTO DE TRÂNSITO DO PARANÁ (DETRAN/PR), Informe Estatístico. Curitiba, 2008.

FREITAS, C. Internações e óbitos e sua relação com a poluição atmosférica em São Paulo, 1993 a 1997.Rev. Saúde Pública, São Paulo, v. 38, n. 6, p. 751 - 757, 2004.

GRIZZLE, J. E.; STARMER, C. F.; KOCH, G. G. Analyses of categorical data by linear models. Biometrics, University of North Carolina, Chapel Hill, U.S.A. v. 25, p. 489 - 504, 1969.

HASTIE, T. J.; TIBSHIRANI, R. J. Generalized Additive Models. London: Chapman and Hall, 1990.

INSTITUTO AMBIENTAL DO PARANÁ (IAP). Relatório de Avaliação da Qualidade do Ar 2008, Curitiba, 2008.

INSTITUTO BRASILEIRO DE GEOGRAFIA E ESTATÍSTICA (IBGE). Censo 2010, 2010.

INSTITUTO DE PESQUISA E PLANEJAMENTO DE CURITIBA (IPPUC). Caracterização Geopolítica dos Municípios da Região Metropolitana de Curitiba, 2002.

NASCIMENTO, L. F. C.; PEREIRA, L. A. A.; BRAGA, A. L. F.; MÓDOLO, M. C. C.; CARVALHO JR, J. A. Efeitos da poluição atmosférica na saúde infantil em São José dos Campos, SP.Rev. Saúde Pública [online]. v. 40, n. 1, 2006.

NELDER J. A.; WEDDERBURN R. W. M. Generalized Linear Models. J R Stat Soc A, Londres, n. 135 , p. 370 - 384, 1972.

NICHOL, K. L.; NORDIN, J. D.; NELSON, D. B.; MULLOOLY, J. P.; HAK, E.Effectiveness of influenza vaccine in the community-dwelling elderly. New England Journal of Medicine. n. 357, p. 1373 - 1381, 2007.

OEI, Organização dos Estados Iberoamericanos. Relatório Técnico 2006, 2006.

R DEVELOPMENT CORE TEAM. R: A language and environment for statistical computing. $R$ Foundation for Statistical Computing, Vienna, Austria, 2007. ISBN 3-900051-07-0, URL http://www.Rproject.org.

RIGBY, R. A.; STASINOPOULOS, D. M. The GAMLSS project: a flexible approach to statistical modeling. In.: NEW TRENDS IN STATISTICAL MODELLING: Proceedings of the 16th International Workshop on Statistical Modelling, ed:Klein, B. and Korsholm, L, 249 - 256, 2001. Odense, Denmark.

Generalized Additive Models for Location, Scale and Shape, (with discussion). Appl. Statist., Leeds, v. 54, p. 507 - 554, 2005.

AKANTZILIOTOU C. A framework for modelling overdispersed count data, including the Poisson-shifted generalized inverse Gaussian distribution. Computational Statistics and Data Analysis, Los Angeles, v. 53, p. 381 - 393, 2008.

WANG, C. S.; WANG, S. T. Reducing major cause-specific hospitalization rates and shortening stays after influenza vaccination. Clinical Infectious Diseases, Boston, v. 1, n. 39, p. 1604 - 1610, 2004.

WHO. The World Report 2004. Geneva: WHO, 2004. 
